MATHEMATICS OF COMPUTATION

Volume 71, Number 238, Pages 507-525

S $0025-5718(01) 01361-8$

Article electronically published on November 19, 2001

\title{
CONVERGENCE OF THE MULTIGRID $V$-CYCLE ALGORITHM FOR SECOND-ORDER BOUNDARY VALUE PROBLEMS WITHOUT FULL ELLIPTIC REGULARITY
}

\author{
SUSANNE C. BRENNER
}

\begin{abstract}
The multigrid $V$-cycle algorithm using the Richardson relaxation scheme as the smoother is studied in this paper. For second-order elliptic boundary value problems, the contraction number of the $V$-cycle algorithm is shown to improve uniformly with the increase of the number of smoothing steps, without assuming full elliptic regularity. As a consequence, the $V$-cycle convergence result of Braess and Hackbusch is generalized to problems without full elliptic regularity.
\end{abstract}

\section{INTRODUCTION}

In this paper we consider the effect of the regularity of a second-order elliptic boundary value problem on the asymptotic behavior of the contraction number of a $V$-cycle multigrid algorithm with respect to the number of smoothing steps.

Let $\Omega$ be a polygonal domain in $\mathbb{R}^{2}$ with reentrant corners. Consider the variational problem of finding $u \in H_{0}^{1}(\Omega)$ such that

$$
a(u, v)=F(v) \quad \forall v \in H_{0}^{1}(\Omega),
$$

where $F \in H^{-1}(\Omega)$, the dual space of $H_{0}^{1}(\Omega)$, and

$$
a(u, v)=\int_{\Omega}[p(x) \nabla u \cdot \nabla v+r(x) u v] d x \quad \forall u, v \in H^{1}(\Omega) .
$$

We assume that $p(x)$ and $r(x)$ are $C^{1}$ on $\bar{\Omega}, p(x)>0$ on $\bar{\Omega}$, and $r(x) \geq 0$ on $\bar{\Omega}$.

It is clear that

$$
\left|a\left(v_{1}, v_{2}\right)\right| \leq C\left\|v_{1}\right\|_{H^{1}(\Omega)}\left\|v_{2}\right\|_{H^{1}(\Omega)} \quad \forall v_{1}, v_{2} \in H^{1}(\Omega),
$$

and the Poincaré inequality implies that

$$
a(v, v) \geq c\|v\|_{H^{1}(\Omega)}^{2} \quad \forall v \in H_{0}^{1}(\Omega) .
$$

Here $C$ and $c$ are positive constants which depend on $\Omega$ and the coefficients $p(x)$ and $r(x)$.

Received by the editor August 18, 1999 and, in revised form, October 27, 1999 and July 10, 2000 .

2000 Mathematics Subject Classification. Primary 65N55, 65N30.

Key words and phrases. $V$-cycle multigrid algorithm, second-order boundary value problems without full elliptic regularity.

This work was supported in part by the National Science Foundation under Grant Nos. DMS96-00133 and DMS-00-74246. 
The estimates (1.3)-(1.4) and the Riesz representation theorem imply (cf. 16] 14]) that (1.1) is uniquely solvable and

$$
\|u\|_{H^{1}(\Omega)} \lesssim\|F\|_{H^{-1}(\Omega)} .
$$

In order to avoid the proliferation of constants, we henceforth use the notation $A \lesssim B($ or $B \gtrsim A)$ to represent the inequality $A \leq$ constant $\times B$, where the constant is positive and independent of all the variables in the inequality, and it is always assumed to be mesh-independent (i.e., it is independent of mesh sizes and mesh levels). The notation $A \approx B$ is equivalent to the statement that $A \lesssim B$ and $B \lesssim A$.

Because of the presence of reentrant corners, the solution of (1.1) does not have $H^{2}(\Omega)$ regularity for $F \in L_{2}(\Omega)$. Instead (cf. Corollary 5.12 and Section 14 of [18], and also the related work in [21, 27]), there exists a number $\alpha$ satisfying

$$
\frac{1}{2}<\alpha<1
$$

such that $u \in H^{1+\alpha}(\Omega) \cap H_{0}^{1}(\Omega)$ for $F \in H^{-1+\alpha}(\Omega)$, and the following regularity estimate holds:

$$
\|u\|_{H^{1+\alpha}(\Omega)} \lesssim\|F\|_{H^{-1+\alpha}(\Omega)} .
$$

Let $\mathcal{T}_{1}$ be a triangulation of $\Omega$, and the triangulations $\mathcal{T}_{k}, k=2,3, \ldots$, be obtained from $\mathcal{T}_{k-1}$ by connecting midpoints. The $\mathrm{P}_{1}$ finite element spaces associated with the triangulations $\mathcal{T}_{k}$ will be denoted by $V_{k}$. The $k^{\text {th }}$ level discrete problem for (1.1) is to find $u_{k} \in V_{k}$ such that

$$
a\left(u_{k}, v\right)=F(v) \quad \forall v \in V_{k} .
$$

On each level we introduce a discrete inner product

$$
\left(v_{1}, v_{2}\right)_{k}=h_{k}^{2} \sum_{p \in \mathcal{V}_{k}} v_{1}(p) v_{2}(p) \quad \forall v_{1}, v_{2} \in V_{k},
$$

where $\mathcal{V}_{k}$ is the set of all the internal vertices of $\mathcal{T}_{k}$. We can then represent the variational form $a(\cdot, \cdot)$ on $V_{k} \times V_{k}$ by the operator $A_{k}: V_{k} \longrightarrow V_{k}$ defined by

$$
\left(A_{k} v_{1}, v_{2}\right)_{k}=a\left(v_{1}, v_{2}\right) \quad \forall v_{1}, v_{2} \in V_{k} .
$$

Note that $A_{k}$ is symmetric positive definite with respect to $(\cdot, \cdot)_{k}$.

The discrete problem (1.7) can be rewritten as

$$
A_{k} u_{k}=f_{k},
$$

where $f_{k} \in V_{k}$ is defined by

$$
\left(f_{k}, v\right)_{k}=F(v) \quad \forall v \in V_{k} .
$$

The $V$-cycle multigrid algorithm (cf. [23, 25, 5] [10]) is an iterative solver for equations of the form (1.10). Given $g \in V_{k}$ and initial guess $z_{0} \in V_{k}$, it produces $M G_{\mathcal{V}}\left(k, g, z_{0}, m_{1}, m_{2}\right)$ as an approximate solution for the equation

$$
A_{k} z=g,
$$

where $m_{1}$ (resp. $m_{2}$ ) is the number of pre-smoothing (resp. post-smoothing) steps. The following are the known results concerning the convergence of the $V$-cycle multigrid algorithm. 
In the case of full elliptic regularity where $\alpha=1$, Braess and Hackbusch 22, 4, 23 . (cf. also [1, 25, 7]) proved that there exists a positive mesh-independent constant $C$ such that

$$
\begin{aligned}
\| z- & M G_{\mathcal{V}}\left(k, g, z_{0}, m_{1}, m_{2}\right) \|_{a} \\
\leq & {\left[\frac{C}{C+\left[\max \left(m_{1}, 1\right) \max \left(m_{2}, 1\right)\right]^{1 / 2}}\right]\left\|z-z_{0}\right\|_{a} }
\end{aligned}
$$

for $m_{1}+m_{2} \geq 1$, where the energy norm $\|\cdot\|_{a}$ is defined by

$$
\|v\|_{a}=\sqrt{a(v, v)} \quad \forall v \in H_{0}^{1}(\Omega) .
$$

For the case where $\alpha<1$, Zhang [33] and Bramble and Pasciak [8, 9] (cf. also 31, 32, 20, 11, 28]) showed that there exists a positive constant $\delta<1$, independent of the meshes and the number of smoothing steps, such that

$$
\left\|z-M G_{\mathcal{V}}\left(k, g, z_{0}, m_{1}, m_{2}\right)\right\|_{a} \leq \delta\left\|z-z_{0}\right\|_{a} .
$$

Note that, in contrast to (1.13), the estimate (1.15) does not indicate that the contraction number of the $V$-cycle algorithm decreases with the increase of the number of smoothing steps.

In this paper we develop a new additive approach to the convergence of the $V$-cycle algorithm and obtain the following estimate (cf. Lemma 6.8):

$$
\left\|z-M G_{\mathcal{V}}\left(k, g, z_{0}, 0, m\right)\right\|_{a} \lesssim m^{-\alpha / 2}\left\|z-z_{0}\right\|_{a}
$$

for $m$ sufficiently large. Combining (1.15) and (1.16), we have (cf. Theorem 6.9)

$$
\begin{aligned}
\| z- & M G_{\mathcal{V}}\left(k, g, z_{0}, m_{1}, m_{2}\right) \|_{a} \\
\leq & {\left[\frac{C}{C+\left[\max \left(m_{1}, 1\right) \max \left(m_{2}, 1\right)\right]^{\alpha / 2}}\right]\left\|z-z_{0}\right\|_{a} }
\end{aligned}
$$

for $m_{1}+m_{2} \geq 1$, where the positive constant $C$ is mesh-independent. In other words, the estimate (1.13) is generalized to boundary value problems without full elliptic regularity.

Remark 1.1. The new result (1.17) is obtained only for the $V$-cycle algorithm using the Richardson relaxation scheme as the smoother (cf. Section 21), while the estimates (1.13) and (1.15) are valid for many other smoothers. The generalization of (1.17) to other smoothers will be investigated in future work.

The rest of the paper is organized as follows. The multigrid $V$-cycle algorithm with the Richardson relaxation scheme as the smoother is described in Section 2 Tools for the analysis of the multigrid $V$-cycle algorithm are developed in Sections 3 .5. The estimates (1.16) and (1.17) are then proved in Section 6

\section{A multigrid $V$-CyCle ALgORIthm}

In this section we describe the $V$-cycle multigrid algorithm with Richardson relaxation as the smoother. First we note that the $\mathrm{P}_{1}$ finite element spaces $V_{k}$ are nested, i.e.,

$$
V_{1} \subset V_{2} \subset \cdots \subset H_{0}^{1}(\Omega),
$$

and the mesh sizes $h_{k}=\max _{T \in \mathcal{T}_{k}} \operatorname{diam} T$ are related by

$$
h_{k}=2 h_{k+1} \quad \text { for } \quad k=1,2,3, \ldots
$$


It is also easy to see that the mesh-dependent inner product defined by (1.8) satisfies

$$
(v, v)_{k} \approx\|v\|_{L_{2}(\Omega)}^{2} \quad \forall v \in V_{k},
$$

and then (1.3) and a standard inverse estimate (cf. [16, 14]) imply that the spectral radius $\rho\left(A_{k}\right)$ satisfies

$$
\rho\left(A_{k}\right) \lesssim h_{k}^{-2}
$$

The computation of $M G_{\mathcal{V}}\left(k, g, z_{0}, m_{1}, m_{2}\right)$ is defined recursively as follows.

Algorithm 2.1. For $k=1$, we define

$$
M G_{\mathcal{V}}\left(1, g, z_{0}, m_{1}, m_{2}\right)=A_{1}^{-1} g .
$$

For $k \geq 2$, we obtain $M G_{\mathcal{V}}\left(k, g, z_{0}, m_{1}, m_{2}\right)$ in three steps.

1. (Pre-Smoothing) For $j=1,2, \ldots, m_{1}$, compute $z_{j}$ by

$$
z_{j}=z_{j-1}+\frac{1}{\Lambda_{k}}\left(g-A_{k} z_{j-1}\right) .
$$

2. (Coarse Grid Correction) Compute $z_{m_{1}+1}$ by

$$
z_{m_{1}+1}=z_{m_{1}}+M G_{\mathcal{V}}\left(k-1, I_{k}^{k-1}\left(g-A_{k} z_{m_{1}}\right), 0, m_{1}, m_{2}\right) .
$$

3. (Post-Smoothing) For $j=m_{1}+2, \ldots, m_{1}+m_{2}+1$, compute $z_{j}$ by

$$
z_{j}=z_{j-1}+\frac{1}{\Lambda_{k}}\left(g-A_{k} z_{j-1}\right) \text {. }
$$

Finally we set $M G_{\mathcal{V}}\left(k, g, z_{0}, m_{1}, m_{2}\right)$ to be $z_{m_{1}+m_{2}+1}$.

In 2.6 and (2.8), the number $\Lambda_{k}$ satisfies

$$
\rho\left(A_{k}\right) \leq \Lambda_{k}
$$

and the intergrid transfer operator $I_{k}^{k-1}: V_{k} \longrightarrow V_{k-1}$ in (2.7) is defined by

$$
\left(I_{k}^{k-1} v, w\right)_{k-1}=(v, w)_{k} \quad \forall v \in V_{k}, w \in V_{k-1} .
$$

In view of (2.4), we can always take $\Lambda_{k}=C h_{k}^{-2}$, where the positive constant $C$ is mesh-independent.

Next we recall briefly some well-known formulas that describe the errors of $V$ cycle algorithms. More details can be found in [26, 23, 5, 10].

Let $E_{k, m_{1}, m_{2}}: V_{k} \longrightarrow V_{k}$ be the operator connecting the initial error and the final error of the multigrid $V$-cycle algorithm applied to (1.12), i.e.,

$$
E_{k, m_{1}, m_{2}}\left(z-z_{0}\right)=z-M G_{\mathcal{V}}\left(k, g, z_{0}, m_{1}, m_{2}\right)
$$

The operator $E_{k, m_{1}, m_{2}}$ can be described in terms of the Ritz projection operators $P_{k}: H_{0}^{1}(\Omega) \longrightarrow V_{k}$ defined by

$$
a\left(P_{k} \zeta, v\right)=a(\zeta, v) \quad \forall \zeta \in H_{0}^{1}(\Omega), v \in V_{k},
$$

and the operators $R_{k}: V_{k} \longrightarrow V_{k}$ defined by

$$
R_{k}=I d_{k}-\frac{1}{\Lambda_{k}} A_{k},
$$

where $I d_{k}$ is the identity operator on $V_{k}$.

Remark 2.2. It follows immediately from (1.14) and (2.12) that

$$
\begin{aligned}
\left\|P_{k} \zeta\right\|_{a} & \leq\|\zeta\|_{a} & & \forall \zeta \in H_{0}^{1}(\Omega), \\
\left\|\zeta-P_{k} \zeta\right\|_{a} & =\inf _{v \in V_{k}}\|\zeta-v\|_{a} & & \forall \zeta \in H_{0}^{1}(\Omega) .
\end{aligned}
$$


Note that (1.9), (2.10) and (2.12) imply

$$
I_{k}^{k-1} A_{k} v=A_{k-1} P_{k} v \quad \forall v \in V_{k} .
$$

Using (2.11), (2.13) and (2.16), we can express the effects of (2.6) $-(2.8)$ as

$$
\begin{aligned}
z-z_{m_{1}} & =R_{k}^{m_{1}}\left(z-z_{0}\right), \\
z-z_{m_{1}+1} & =\left(I d_{k}-P_{k-1}+E_{k-1, m_{1}, m_{2}} P_{k-1}\right)\left(z-z_{m_{1}}\right), \\
z-z_{m_{1}+m_{2}+1} & =R_{k}^{m_{2}}\left(z-z_{m_{1}+1}\right) .
\end{aligned}
$$

Comparing (2.11) and (2.17), we obtain the recursive relation

$$
\begin{aligned}
& E_{k, m_{1}, m_{2}} v=R_{k}^{m_{2}}\left(I d_{k}-P_{k-1}+E_{k-1, m_{1}, m_{2}} P_{k-1}\right) R_{k}^{m_{1}} v \\
& \forall v \in V_{k}, k \geq 2,
\end{aligned}
$$

and of course we also have (cf. (2.5))

$$
E_{1, m_{1}, m_{2}} v=0 \quad \forall v \in V_{1} .
$$

In particular the operators $E_{k, m}=E_{k, 0, m}$ and $E_{k, m}^{*}=E_{k, m, 0}$ satisfy

$$
\begin{array}{ll}
E_{k, m} v=R_{k}^{m}\left(I d_{k}-P_{k-1}+E_{k-1, m} P_{k-1}\right) v & \forall v \in V_{k}, k \geq 2, \\
E_{1, m} v=0 & \forall v \in V_{1}, \\
E_{k, m}^{*} v=\left(I d_{k}-P_{k-1}+E_{k-1, m}^{*} P_{k-1}\right) R_{k}^{m} v & \forall v \in V_{k}, k \geq 2, \\
E_{1, m}^{*} v=0 & \forall v \in V_{1} .
\end{array}
$$

The following well-known relations (cf. 26, 23]) can be derived by mathematical induction using (2.18)-(2.23):

$$
\begin{aligned}
a\left(E_{k, m} v_{1}, v_{2}\right) & =a\left(v_{1}, E_{k, m}^{*} v_{2}\right) & & \forall v_{1}, v_{2} \in V_{k}, \\
E_{k, m_{1}, m_{2}} & =E_{k, m_{2}} E_{k, m_{1}}^{*} & & \text { for } \quad k=1,2, \ldots .
\end{aligned}
$$

The estimate (1.16) can be rewritten as

$$
\left\|E_{k, m} v\right\|_{a} \lesssim m^{-(\alpha / 2)}\|v\|_{a} \quad \forall v \in V_{k} .
$$

We will establish (2.26) in Section 6 using the following additive expression for $E_{k, m}$, which follows immediately from (2.20) and (2.21).

$$
E_{k, m} v=\sum_{j=2}^{k} R_{k}^{m} R_{k-1}^{m} \cdots R_{j}^{m}\left(P_{j}-P_{j-1}\right) v \quad \forall v \in V_{k} .
$$

Remark 2.3. The operators $E_{k, m}(k \geq 2)$ can also be written as (cf. [5, 10])

$$
E_{k, m} v=R_{k}^{m}\left(I d_{k}-P_{k-1}+R_{k-1}^{m} P_{k-1}\right) \cdots\left(I d_{k}-P_{2}+R_{2}^{m} P_{2}\right)\left(I d_{k}-P_{1}\right) v
$$

for $v \in V_{k}$. This multiplicative representation of $E_{k, m}$ plays a key role in the proofs of many of the known $V$-cycle convergence results. Using (2.27) instead of (2.28) is the point of departure for the new approach to $V$-cycle convergence developed in this paper. 


\section{Consequences of the elliptic Regularity}

First we note that (1.5) and (1.6) imply a scale of elliptic regularity estimates.

Following the notation in 30 , 21], we define, for $s \geq 0$, the space $\tilde{H}^{s}(\Omega)=$ $\left\{v \in H^{s}(\Omega): \tilde{v} \in H^{s}\left(\mathbb{R}^{2}\right)\right\}$, where $\tilde{v}$ is the trivial extension of $v$ to $\mathbb{R}^{2}$, and $\|v\|_{\tilde{H}^{s}(\Omega)}=\|\tilde{v}\|_{H^{s}\left(\mathbb{R}^{2}\right)}$. The spaces $\tilde{H}^{s}(\Omega)$ for $s \geq 0$ form a scale of interpolation spaces. Moreover, for $s-\frac{1}{2} \notin \mathbb{Z}$, the space $\tilde{H}^{s}(\Omega)$ coincides with $H_{0}^{s}(\Omega)$, and the norms $\|\cdot\|_{\tilde{H}^{s}(\Omega)}$ and $\|\cdot\|_{H^{s}(\Omega)}$ are equivalent on $\tilde{H}^{s}(\Omega)=H_{0}^{s}(\Omega)$. Consequently, for $s \geq 0$ and $s-\frac{1}{2} \notin \mathbb{Z}$, we have $H^{-s}(\Omega)=\left[H_{0}^{s}(\Omega)\right]^{\prime}=\left[\tilde{H}^{s}(\Omega)\right]^{\prime}$ with equivalent norms.

Let $0 \leq s \leq \alpha$ and $u \in H_{0}^{1}(\Omega)$ satisfy (1.1) for $F \in\left[\tilde{H}^{1-s}(\Omega)\right]^{\prime}$. The interpolation theory of Sobolev spaces (cf. [30, 3]) and the estimates (1.5) and (1.6) imply that $u \in H^{1+s}(\Omega)$ and

$$
\|u\|_{H^{1+s}(\Omega)} \lesssim\|F\|_{\left[\tilde{H}^{1-s}(\Omega)\right]^{\prime}} .
$$

It follows from (13.1) that $T: H^{1+s}(\Omega) \cap H_{0}^{1}(\Omega) \longrightarrow\left[\tilde{H}^{1-s}(\Omega)\right]^{\prime}$ defined by

$$
T \phi=-\nabla \cdot(p(x) \nabla \phi)+r(x) \phi
$$

is an isomorphism for $0 \leq s \leq \alpha$; i.e., $T$ is one-to-one, onto and the following estimate holds:

$$
\|T \phi\|_{\left[\tilde{H}^{1-s}(\Omega)\right]^{\prime}} \approx\|\phi\|_{H^{1+s}(\Omega)} \quad \forall \phi \in H^{1+s}(\Omega) \cap H_{0}^{1}(\Omega) .
$$

Moreover, by a density argument, the variational form $a(\cdot, \cdot)$ has a unique extension from $H_{0}^{1}(\Omega) \times H_{0}^{1}(\Omega)$ to $\left[H^{1+s}(\Omega) \cap H_{0}^{1}(\Omega)\right] \times \tilde{H}^{1-s}(\Omega)$ such that

$$
a(\phi, \psi)=(T \phi)(\psi) \quad \forall \phi \in H^{1+s}(\Omega) \cap H_{0}^{1}(\Omega), \psi \in \tilde{H}^{1-s}(\Omega) .
$$

The Sobolev spaces $H^{1+s}(\Omega) \cap H_{0}^{1}(\Omega)$ and $\tilde{H}^{1-s}(\Omega)$ satisfy a duality relation with respect to the extended variational form $a(\cdot, \cdot)$, as stated in the next lemma.

Lemma 3.1. The following estimates hold for $0 \leq s \leq \alpha$ :

$$
\begin{array}{ll}
\|\phi\|_{H^{1+s}(\Omega)} \approx \sup _{\substack{\psi \in \tilde{H}^{1-s}(\Omega) \\
\psi \neq 0}} \frac{a(\phi, \psi)}{\|\psi\|_{\tilde{H}^{1-s}(\Omega)}} & \forall \phi \in H^{1+s}(\Omega) \cap H_{0}^{1}(\Omega), \\
\|\psi\|_{\tilde{H}^{1-s}(\Omega)} \approx \sup _{\substack{\phi \in H^{1+s}(\Omega) \cap H_{0}^{1}(\Omega) \\
\phi \neq 0}} \frac{a(\phi, \psi)}{\|\phi\|_{H^{1+s}(\Omega)}} & \forall \psi \in \tilde{H}^{1-s}(\Omega) .
\end{array}
$$

Proof. The estimates (3.4) and (3.5) follow immediately from (3.2), (3.3) and the standard duality formulas

$$
\begin{array}{rlrl}
\|F\|_{\left[\tilde{H}^{1-s}(\Omega)\right]^{\prime}} & =\sup _{\substack{\psi \in \tilde{H}^{1-s}(\Omega) \\
\psi \neq 0}} \frac{F(\psi)}{\|\psi\|_{\tilde{H}^{1-s}(\Omega)}} & \forall F \in\left[\tilde{H}^{1-s}(\Omega)\right]^{\prime}, \\
\|\psi\|_{\tilde{H}^{1-s}(\Omega)} & =\sup _{\substack{F \in\left[\tilde{H}^{1-s}(\Omega)\right]^{\prime} \\
F \neq 0}} \frac{F(\psi)}{\|F\|_{\left[\tilde{H}^{1-s}(\Omega)\right]^{\prime}}} \quad \forall \psi \in \tilde{H}^{1-s}(\Omega) .
\end{array}
$$

Lemma 3.1 can be used to measure the approximation properties of the finite element spaces $V_{k}$ in lower order Sobolev norms. 
Lemma 3.2. The following estimate holds:

$$
\left\|\zeta-P_{k} \zeta\right\|_{\tilde{H}^{1-s}(\Omega)} \lesssim h_{k}^{s}\|\zeta\|_{a} \quad \forall 0 \leq s \leq \alpha, \zeta \in H_{0}^{1}(\Omega) .
$$

Proof. There exists an operator (cf. [17 29]) $\mathcal{I}_{k}: H_{0}^{1}(\Omega) \longrightarrow V_{k}$ such that

$$
\left\|\phi-\mathcal{I}_{k} \phi\right\|_{H^{1}(\Omega)} \lesssim h_{k}^{s}\|\phi\|_{H^{1+s}(\Omega)} \quad \forall \phi \in H^{1+s}(\Omega) \cap H_{0}^{1}(\Omega) .
$$

By (1.3), (2.15) and (3.7) we have

$$
\left\|\phi-P_{k} \phi\right\|_{a} \lesssim h_{k}^{s}\|\phi\|_{H^{1+s}(\Omega)} \quad \forall \phi \in H^{1+s}(\Omega) \cap H_{0}^{1}(\Omega) .
$$

Using (1.14), (2.12), (3.5), and (3.8), we can establish (3.6) as follows:

$$
\begin{aligned}
\left\|\zeta-P_{k} \zeta\right\|_{\tilde{H}^{1-s}(\Omega)} & \approx \sup _{\substack{\phi \in H^{1+s}(\Omega) \cap H_{0}^{1}(\Omega) \\
\phi \neq 0}} \frac{a\left(\phi, \zeta-P_{k} \zeta\right)}{\|\phi\|_{H^{1+s}(\Omega)}} \\
& =\sup _{\substack{\phi \in H^{1+s}(\Omega) \cap H_{0}^{1}(\Omega) \\
\phi \neq 0}} \frac{a\left(\phi-P_{k} \phi, \zeta\right)}{\|\phi\|_{H^{1+s}(\Omega)}} \\
& \lesssim \sup _{\substack{\phi \in H^{1+s}(\Omega) \cap H_{0}^{1}(\Omega) \\
\phi \neq 0}} \frac{\left\|\phi-P_{k} \phi\right\|_{a}\|\zeta\|_{a}}{\|\phi\|_{H^{1+s}(\Omega)}} \lesssim h_{k}^{s}\|\zeta\|_{a} .
\end{aligned}
$$

\section{Mesh DePEndent NORMS}

Following [2] we define the mesh dependent norms $\|\cdot\|_{s, k}(s \in \mathbb{R})$ by

$$
\|v\|_{s, k}=\sqrt{\left(A_{k}^{s} v, v\right)_{k}} \quad \forall v \in V_{k} .
$$

It is clear from (1.3), (1.4), (1.9), (1.14), (2.3), (2.4), and (4.1) that

$$
\begin{array}{rlrl}
\|v\|_{1, k} & =\|v\|_{a} \approx\|v\|_{H^{1}(\Omega)} & & \forall v \in V_{k}, \\
\|v\|_{0, k} \approx\|v\|_{L_{2}(\Omega)} & & \forall v \in V_{k}, \\
\|v\|_{s, k} & \lesssim h_{k}^{t-s}\|v\|_{t, k} & & \forall v \in V_{k}, 0 \leq t \leq s \leq 2 .
\end{array}
$$

It also follows immediately from (4.1) that

$$
\|v\|_{1+t, k}=\sup _{\substack{w \in V_{k} \\ w \neq 0}} \frac{a(v, w)}{\|w\|_{1-t, k}} \quad \forall v \in V_{k}, t \in \mathbb{R} .
$$

In particular, we have the following generalized Cauchy-Schwarz inequality:

$$
\left|a\left(v_{1}, v_{2}\right)\right| \leq\left\|v_{1}\right\|_{1+t, k}\left\|v_{2}\right\|_{1-t, k} \quad \forall v_{1}, v_{2} \in V_{k}, t \in \mathbb{R} .
$$

These mesh dependent norms are related to the Sobolev norms, in fact (cf. [2, 12]),

$$
\|v\|_{s, k} \approx\|v\|_{\tilde{H}^{s}(\Omega)} \quad \forall v \in V_{k}, 0 \leq s \leq 1 .
$$

The following smoothing properties of $R_{k}$ are well known (cf. [2, 23]):

$$
\begin{aligned}
\left\|R_{k}^{m} v\right\|_{s, k} & \lesssim\left(h_{k} \sqrt{m}\right)^{t-s}\|v\|_{t, k} & & \forall v \in V_{k}, 0 \leq t \leq s \leq 2, \\
\left\|R_{k} v\right\|_{s, k} & \leq\|v\|_{s, k} & & \forall v \in V_{k}, s \in \mathbb{R} .
\end{aligned}
$$

The combined effect of the smoothing and approximation properties is given in the next lemma. 
Lemma 4.1. The following estimates hold for $0 \leq s \leq \alpha$ :

$$
\left\|R_{k}^{m}\left(I d_{k}-P_{k-1}\right) v\right\|_{1-s, k} \lesssim m^{(s-\alpha) / 2} h_{k}^{s}\|v\|_{a} \quad \forall v \in V_{k} .
$$

Proof. Combining (2.2), (3.6), (4.2), (4.7) and (4.8), we have

$$
\begin{aligned}
\left\|R_{k}^{m}\left(I d_{k}-P_{k-1}\right) v\right\|_{1-s, k} & \lesssim\left(h_{k} \sqrt{m}\right)^{s-\alpha}\left\|v-P_{k-1} v\right\|_{1-\alpha, k} \\
& \approx\left(h_{k} \sqrt{m}\right)^{s-\alpha}\left\|v-P_{k-1} v\right\|_{\tilde{H}^{1-\alpha}(\Omega)} \\
& \lesssim\left(h_{k} \sqrt{m}\right)^{s-\alpha} h_{k-1}^{\alpha}\|v\|_{a} \lesssim m^{(s-\alpha) / 2} h_{k}^{s}\|v\|_{a} .
\end{aligned}
$$

Let $\beta$ be a fixed number satisfying $0<\beta<\frac{1}{2}$. Note that (cf. [13, 30]) $V_{k} \subset$ $H_{0}^{1+\beta}(\Omega)=H^{1+\beta}(\Omega) \cap H_{0}^{1}(\Omega)$. Later on we will need the relation between $\|\cdot\|_{1+\beta, k}$ and $\|\cdot\|_{H^{1+\beta}(\Omega)}$ stated in the next lemma, whose proof uses the error estimate (cf. [16, 19, 14])

$$
\left\|\zeta-\Pi_{k} \zeta\right\|_{a} \lesssim h_{k}^{\beta}\|\zeta\|_{H^{1+\beta}(\Omega)} \quad \forall \zeta \in H_{0}^{1+\beta}(\Omega)
$$

for the nodal interpolation operator $\Pi_{k}$, and also (cf. [13]) the estimate

$$
\left\|\Pi_{k} \zeta\right\|_{H^{1+\beta}(\Omega)} \lesssim\|\zeta\|_{H^{1+\beta}(\Omega)} \quad \forall \zeta \in H_{0}^{1+\beta}(\Omega) .
$$

Lemma 4.2. The following estimate holds:

$$
\|v\|_{1+\beta, k} \approx\|v\|_{H^{1+\beta}(\Omega)} \quad \forall v \in V_{k} .
$$

Proof. It follows from (3.4), (4.5) and (4.7) that

$$
\begin{aligned}
\|v\|_{1+\beta, k}=\sup _{\substack{w \in V_{k} \\
w \neq 0}} \frac{a(v, w)}{\|w\|_{1-\beta, k}} & \approx \sup _{\substack{w \in V_{k} \\
w \neq 0}} \frac{a(v, w)}{\|w\|_{\tilde{H}^{1-\beta}(\Omega)}} \\
& \lesssim \sup _{\substack{\psi \in \tilde{H}^{1-\beta}(\Omega) \\
\psi \neq 0}} \frac{a(v, \psi)}{\|\psi\|_{\tilde{H}^{1-\beta}(\Omega)}} \lesssim\|v\|_{H^{1+\beta}(\Omega)} \quad \forall v \in V_{k} .
\end{aligned}
$$

To prove the converse, we first observe that, since $V_{k} \subset H_{0}^{1+\beta}(\Omega)$, the Ritz projection operator $P_{k}$ can be extended to $\tilde{H}^{1-\beta}(\Omega)$, by (3.3), so that

$$
a\left(v, P_{k} \psi\right)=a(v, \psi) \quad \forall v \in V_{k}, \psi \in \tilde{H}^{1-\beta}(\Omega) .
$$

Let $\phi \in H_{0}^{1+s}(\Omega)$ be arbitrary. We find, by (3.4), (3.8), (4.2), (4.11), (4.12), (4.14) and standard inverse estimates,

$$
\begin{aligned}
a\left(\phi, P_{k} \psi\right) & =a\left(P_{k} \phi-\Pi_{k} \phi, \psi\right)+a\left(\Pi_{k} \phi, \psi\right) \\
& \lesssim\|\psi\|_{\tilde{H}^{1-\beta}(\Omega)}\left(\left\|P_{k} \phi-\Pi_{k} \phi\right\|_{H^{1+\beta}(\Omega)}+\left\|\Pi_{k} \phi\right\|_{H^{1+\beta}(\Omega)}\right) \\
& \lesssim\|\psi\|_{\tilde{H}^{1-\beta}(\Omega)}\left(h^{-\beta}\left\|P_{k} \phi-\Pi_{k} \phi\right\|_{H^{1}(\Omega)}+\|\phi\|_{H^{1+\beta}(\Omega)}\right) \\
& \lesssim\|\psi\|_{\tilde{H}^{1-\beta}(\Omega)}\|\phi\|_{H^{1+\beta}(\Omega)},
\end{aligned}
$$

which, in view of (3.5), implies

$$
\left\|P_{k} \psi\right\|_{\tilde{H}^{1-\beta}(\Omega)} \lesssim\|\psi\|_{\tilde{H}^{1-\beta}(\Omega)} .
$$

Let $v \in V_{k}$ be arbitrary. We have, by (4.6), (4.7), (4.14) and (4.15),

$$
\begin{aligned}
a(v, \psi)=a\left(v, P_{k} \psi\right) & \leq\|v\|_{1+\beta, k}\left\|P_{k} \psi\right\|_{1-\beta, k} \\
& \lesssim\|v\|_{1+\beta, k}\left\|P_{k} \psi\right\|_{\tilde{H}^{1-\beta}(\Omega)} \\
& \lesssim\|v\|_{1+\beta, k}\|\psi\|_{\tilde{H}^{1-\beta}(\Omega)} \quad \forall \psi \in \tilde{H}^{1-\beta}(\Omega),
\end{aligned}
$$

and therefore, in view of (3.4), $\|v\|_{H^{1+\beta}(\Omega)} \lesssim\|v\|_{1+\beta, k} \forall v \in V_{k}$. 


\section{Relations BetWEen MESH DEPENDENT NORMS ON CONSECUTIVE LEVELS}

Our goal in this section is to show that, given any $\theta \in(0,1]$, we have

$$
\begin{aligned}
\|v\|_{1-\beta, k+1}^{2} \leq\left(1+\theta^{2}\right)\|v\|_{1-\beta, k}^{2}+C \theta^{-4} h_{k}^{2 \beta}\|v\|_{a}^{2} & \forall v \in V_{k}, \\
\left\|\Pi_{k-1} v\right\|_{1-\beta, k-1}^{2} \leq\left(1+\theta^{2}\right)\|v\|_{1-\beta, k}^{2}+C \theta^{-4} h_{k}^{2 \beta}\|v\|_{a}^{2} & \forall v \in V_{k},
\end{aligned}
$$

where $\beta$ is the number chosen in Section 4 , and $C$ (and any other constant in this section and the next) is a generic positive constant independent of the meshes and $\theta$, which can take different values at different places. The estimates (5.1) and (5.2) play a key role in the convergence analysis in Section 6. Their proofs involve several lemmas.

Let us first introduce another mesh dependent inner product $((\cdot, \cdot))_{k}$ on $V_{k}$ :

$$
\left(\left(v_{1}, v_{2}\right)\right)_{k}=h_{k}^{2} \sum_{p \in \mathcal{V}_{k}} n(p) v_{1}(p) v_{2}(p) \quad \forall v_{1}, v_{2} \in V_{k},
$$

where the function $n(\cdot)$ is defined by

$$
n(p)=\frac{1}{6} \times\left(\text { the number of triangles in } \mathcal{T}_{k} \text { that have } p \text { as a vertex }\right) .
$$

It is easy to see from (5.4) and the construction of $\mathcal{T}_{k}$ that $n(\cdot)$ is independent of mesh levels, and

$$
n(p)=1 \quad \text { for } \quad p \in \mathcal{V}_{k} \backslash \mathcal{V}_{1}
$$

The operator $\mathcal{A}_{k}: V_{k} \longrightarrow V_{k}$ is defined by

$$
\left(\left(\mathcal{A}_{k} v_{1}, v_{1}\right)\right)_{k}=a\left(v_{1}, v_{2}\right) \quad \forall v_{1}, v_{2} \in V_{k} .
$$

We can then define the corresponding mesh dependent norms by

$$
\|v\|_{s, k}=\sqrt{\left(\left(\mathcal{A}_{k}^{s} v_{1}, v_{2}\right)\right)_{k}} .
$$

Remark 5.1. It is clear that all the properties of $\|\cdot\|$ stated in Section 4 also hold for \|\| . I\|. A $V$-cycle algorithm can also be defined by replacing the inner product $(\cdot, \cdot)_{k}$ with $((\cdot, \cdot))_{k}$ throughout Section 2 The two algorithms are different only by a diagonal preconditioner in the smoothing steps. For the algorithm based on $((\cdot, \cdot))_{k}$, the analysis in Section 6 can be carried out using the simpler estimates (5.8) and (5.11) below.

Our plan is to first prove the (simpler) analogs of (5.1) and (5.2) for the new mesh dependent norms, and then obtain (5.1) and (5.2) through the relation between the norms $\|\cdot\| 1-\beta, k$ and $\|\mid \cdot\| \|_{1-\beta, k}$.

Lemma 5.2. The following estimate holds:

$$
\|v\|_{1-\beta, k+1} \leq\|v\|_{1-\beta, k} \quad \forall v \in V_{k} .
$$

Proof. It is clear from (5.6) and (5.7) that

$$
\|\| v\left\|_{1, k+1}=\right\| v\left\|_{a}=\right\| v\|\|_{1, k} \quad \forall v \in V_{k} .
$$

Let the parents of $q \in \mathcal{V}_{k+1} \backslash \mathcal{V}_{k}$ be denoted by $q^{\prime}$ and $q^{\prime \prime}$, i.e., $q^{\prime}, q^{\prime \prime} \in \mathcal{V}_{k}$ and $q$ is the midpoint between them. Note that each $p \in \mathcal{V}_{k}$ is the parent of exactly 
$6 n(p)$ many vertices in $\mathcal{V}_{k+1} \backslash \mathcal{V}_{k}$. Let $v \in V_{k}$ be arbitrary. Using (2.2), (5.3) and (5.5) we can estimate $\|v\|_{0, k+1}^{2}$ by

$$
\begin{aligned}
\|v\|_{0, k+1}^{2} & =h_{k+1}^{2}\left(\sum_{p \in \mathcal{V}_{k}} n(p)[v(p)]^{2}+\sum_{q \in \mathcal{V}_{k+1} \backslash \mathcal{V}_{k}}[v(q)]^{2}\right) \\
& =h_{k+1}^{2}\left(\sum_{p \in \mathcal{V}_{k}} n(p)[v(p)]^{2}+\sum_{q \in \mathcal{V}_{k+1} \backslash \mathcal{V}_{k}}\left[\frac{v\left(q^{\prime}\right)+v\left(q^{\prime \prime}\right)}{2}\right]^{2}\right) \\
& \leq h_{k+1}^{2}\left(\sum_{p \in \mathcal{V}_{k}} n(p)[v(p)]^{2}+\sum_{q \in \mathcal{V}_{k+1} \backslash \mathcal{V}_{k}} \frac{\left[v\left(q^{\prime}\right)\right]^{2}+\left[v\left(q^{\prime \prime}\right)\right]^{2}}{2}\right) \\
& =h_{k+1}^{2}\left(\sum_{p \in \mathcal{V}_{k}} n(p)[v(p)]^{2}+\sum_{p \in \mathcal{V}_{k}} 6 n(p) \frac{[v(p)]^{2}}{2}\right) \\
& =h_{k}^{2} \sum_{p \in \mathcal{V}_{k}} n(p)[v(p)]^{2}=\|v\|_{0, k}^{2} .
\end{aligned}
$$

Hence we have

$$
\|\| v\left\|_{0, k+1} \leq\right\|\|v\|_{0, k} \quad \forall v \in V_{k} .
$$

The estimate (5.8) follows from (5.9), (5.10) and interpolation between the (real) Hilbert scales $\left(V_{k},\|\| \cdot\|\|_{s, k}\right)$ and $\left(V_{k+1},\|\| \cdot\|\|_{s, k+1}\right)$ (cf. Theorem B.4 in [5]).

Lemma 5.3. There exists a positive constant $C$ such that

$$
\left\|\Pi_{k-1} v\right\|_{1-\beta, k-1}^{2} \leq\left(1+\theta^{2}\right)\|v\|_{1-\beta, k}^{2}+C \theta^{-2} h_{k}^{2 \beta}\|v\|_{a}^{2}
$$

$\forall \theta \in(0,1], v \in V_{k}$.

Proof. We have, by (1.14), (2.2), (4.11), (5.9) and the analog of (4.13) for $\|\cdot\| \|_{1+\beta, k}$,

$$
\begin{aligned}
\|\| \Pi_{k-1} v \|_{1, k-1}^{2} & \leq\left(\|v\|_{a}+\left\|v-\Pi_{k-1} v\right\|_{a}\right)^{2} \\
& \leq\left(1+\theta^{2}\right)\|v\|_{a}^{2}+\left(1+\theta^{-2}\right)\left\|v-\Pi_{k-1} v\right\|_{a}^{2} \\
& \leq\left(1+\theta^{2}\right)\|v\|_{1, k}^{2}+c_{1} \theta^{-2} h_{k}^{2 \beta}\|v\|_{1+\beta, k}^{2} \quad \forall v \in V_{k} .
\end{aligned}
$$

Let the parents of $p \in \mathcal{V}_{k} \backslash \mathcal{V}_{k-1}$ be denoted by $p^{\prime}$ and $p^{\prime \prime}$. Given any $v \in V_{k}$, we have the following elementary estimate:

$$
\begin{aligned}
& \frac{\left[v\left(p^{\prime}\right)\right]^{2}}{2}+\frac{\left[v\left(p^{\prime \prime}\right)\right]^{2}}{2} \\
= & \frac{\left(v(p)+\left[v\left(p^{\prime}\right)-v(p)\right]\right)^{2}}{2}+\frac{\left(v(p)+\left[v\left(p^{\prime \prime}\right)-v(p)\right]\right)^{2}}{2} \\
\leq & \left(1+\theta^{2}\right)[v(p)]^{2}+\left(1+\theta^{-2}\right)\left(\frac{\left[v\left(p^{\prime}\right)-v(p)\right]^{2}+\left[v\left(p^{\prime \prime}\right)-v(p)\right]^{2}}{2}\right) .
\end{aligned}
$$

Summing up (5.13) over all $p \in \mathcal{V}_{k} \backslash \mathcal{V}_{k-1}$, we find

$$
3 \sum_{q \in \mathcal{V}_{k-1}} n(q)[v(q)]^{2} \leq\left(1+\theta^{2}\right) \sum_{p \in \mathcal{V}_{k} \backslash \mathcal{V}_{k-1}}[v(p)]^{2}+c_{2} \theta^{-2}|v|_{H^{1}(\Omega)}^{2} .
$$


It follows from (2.2), (5.3), (5.5), (5.14) and the analogs of (4.2) and (4.4) for $\|\cdot\| \|_{s, k}$ that

$$
\begin{aligned}
\left\|\Pi_{k-1} v\right\|_{0, k-1}^{2} & =h_{k-1}^{2} \sum_{q \in \mathcal{V}_{k-1}} n(q)[v(q)]^{2} \\
& \leq h_{k}^{2}\left[\sum_{q \in \mathcal{V}_{k-1}} n(q)[v(q)]^{2}+\left(1+\theta^{2}\right) \sum_{p \in \mathcal{V}_{k} \backslash \mathcal{V}_{k-1}}[v(p)]^{2}+c_{2} \theta^{-2}|v|_{H^{1}(\Omega)}^{2}\right] \\
& \leq h_{k}^{2}\left[\left(1+\theta^{2}\right) \sum_{p \in \mathcal{V}_{k}} n(p)[v(p)]^{2}+c_{3} \theta^{-2}\|v\|_{1, k}^{2}\right] \\
& \leq\left(1+\theta^{2}\right)\|v\|_{0, k}^{2}+c_{4} \theta^{-2} h_{k}^{2 \beta}\|v\|_{\beta, k}^{2} \quad \forall v \in V_{k} .
\end{aligned}
$$

Let $C=\max \left(c_{1}, c_{4}\right)$. Observe that

$$
\left(1+\theta^{2}\right)\|v\|_{s, k}^{2}+C \theta^{-2} h_{k}^{2 \beta}\|v\|_{s+\beta, k}^{2}=\left\langle\mathcal{A}_{k}^{s} v, v\right\rangle_{\theta, k} \quad \forall v \in V_{k},
$$

where the inner product $\langle\cdot, \cdot\rangle_{\theta, k}$ is defined by

$$
\left\langle v_{1}, v_{2}\right\rangle_{\theta, k}=\left(1+\theta^{2}\right)\left(\left(v_{1}, v_{2}\right)\right)_{k}+C \theta^{-2} h_{k}^{2 \beta}\left(\left(\mathcal{A}_{k}^{\beta} v_{1}, v_{2}\right)\right)_{k} \quad \forall v_{1}, v_{2} \in V_{k} .
$$

Therefore, for each $k$, the spaces $\left(V_{k}, \sqrt{\left(1+\theta^{2}\right)\|\| \cdot\|\|_{s, k}^{2}+C \theta^{-2} h_{k}^{2 \beta}\|\| \cdot\|\|_{s+\beta, k}^{2}}\right)$ form a Hilbert scale.

By interpolating (5.12) and (5.15) between the Hilbert scales $\left(V_{k-1},\|\| \cdot\|\|_{s, k-1}\right)$ and $\left(V_{k}, \sqrt{\left(1+\theta^{2}\right)\|\| \cdot\|\|_{s, k}^{2}+C \theta^{-2} h_{k}^{2 \beta}\|\| \cdot \|_{s+\beta, k}^{2}}\right)$, we have

$$
\|\| \Pi_{k-1} v\left\|_{1-\beta, k-1}^{2} \leq\left(1+\theta^{2}\right)\right\| v\left\|_{1-\beta, k}^{2}+C \theta^{-2} h_{k}^{2 \beta}\right\| v \|_{1, k}^{2} \quad \forall v \in V_{k} .
$$

The estimate (5.11) follows from (5.9) and (5.16).

Remark 5.4. It is important that the interpolation between (real) Hilbert scales is exact (cf. Theorem B.4 in 5]) so that no additional constant appears in front of the term $\left(1+\theta^{2}\right)\|v\|_{1-\beta, k}^{2}$ on the right-hand side of (5.11).

The following lemmas relate the two mesh dependent norms.

Lemma 5.5. There exists a positive constant $C$ such that

$$
\begin{aligned}
\|v\|_{0, k}^{2} \leq\left(1+\theta^{2}\right)\|v\|_{0, k}^{2}+C \theta^{-4} h_{k}^{2}\|v\|_{a}^{2} & \forall \theta \in(0,1], v \in V_{k}, \\
\|v\|_{0, k}^{2} \leq\left(1+\theta^{2}\right)\|v\|_{0, k}^{2}+C \theta^{-4} h_{k}^{2}\|v\|_{a}^{2} & \forall \theta \in(0,1], v \in V_{k} .
\end{aligned}
$$

Proof. We will only establish (5.18) since the proof of (5.17) is completely analogous. Also it suffices to show that

$$
\|v\|_{0, k}^{2} \leq\left(1+C^{\prime} \ell^{-2}\right)\|v\|_{0, k}^{2}+C^{\prime \prime} \ell^{4} h_{k}^{2}\|v\|_{H^{1}(\Omega)}^{2} \quad \forall v \in V_{k}, \ell=1,2, \ldots
$$

Let $D=\operatorname{diam} \Omega$ and

$$
d=\min _{\substack{p, q \in \mathcal{V}_{1} \\ p \neq q}}|p-q|
$$

be the minimum of the distances among the distinct vertices of $\mathcal{T}_{1}$.

It is clear from the analog of (2.3) for \|\|$\cdot\|\|_{0, k}$ that, for $\ell h_{k} \geq \frac{d}{2}$,

$$
\|v\|_{0, k}^{2} \leq c_{1} \ell^{4} h_{k}^{2}\|v\|_{H^{1}(\Omega)}^{2} \quad \forall v \in V_{k},
$$

and (5.19) follows. 


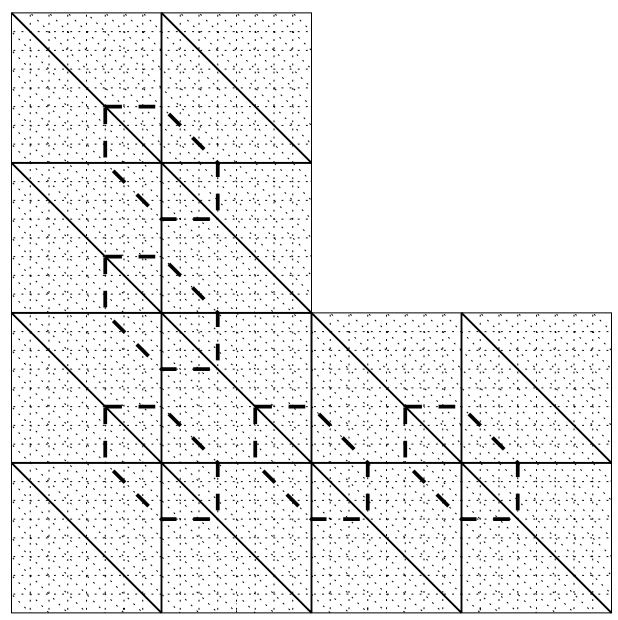

FIGURE 1.

In the case where $\ell h_{k}<\frac{d}{2}$, we define for each $q \in \mathcal{V}_{1}$ the (open) polygon $\Omega_{q}$ whose vertices are those points in $\mathcal{V}_{k}$ that belong to the edges in $\mathcal{T}_{1}$ and which are $\ell$ steps away from $q$. The $\Omega_{q}$ 's for an $L$-shaped domain are depicted in Figure 1, where $k=4$ and $\ell=3$.

It is easy to see that the $\Omega_{q}$ 's are pairwise disjoint and

$$
\operatorname{diam} \Omega \approx \ell h_{k} .
$$

Let $v \in V_{k}$ and $\mathcal{K}=\bigcup_{q \in \mathcal{V}_{1}} \bar{\Omega}_{q}$. By (5.5) we have

$$
h_{k}^{2} \sum_{p \in \mathcal{V}_{k} \backslash \mathcal{K}} n(p)[v(p)]^{2}=h_{k}^{2} \sum_{p \in \mathcal{V}_{k} \backslash \mathcal{K}}[v(p)]^{2} .
$$

Let $q \in \mathcal{V}_{1}$. We can estimate the contribution of the vertices of $\mathcal{T}_{k}$ in $\bar{\Omega}_{q}$ to the left-hand side of (5.19) by

$$
\begin{aligned}
& h_{k}^{2} \sum_{p \in \mathcal{V}_{k} \cap \bar{\Omega}_{q}} n(p)[v(p)]^{2} \\
= & h_{k}^{2} \sum_{p \in \mathcal{V}_{k} \cap \bar{\Omega}_{q}} n(p)\left(v_{q}+\left[v(p)-v_{q}\right]\right)^{2} \\
\leq & \left(1+\ell^{-2}\right) h_{k}^{2}\left(\sum_{p \in \mathcal{V}_{k} \cap \bar{\Omega}_{q}} n(p)\right) v_{q}^{2}+\left(1+\ell^{2}\right) h_{k}^{2} \sum_{p \in \mathcal{V}_{k} \cap \bar{\Omega}_{q}} n(p)\left[v(p)-v_{q}\right]^{2},
\end{aligned}
$$

where the number $v_{q}$ is defined by

$$
v_{q}=\frac{1}{\left|\Omega_{q}\right|} \int_{\Omega_{q}} v d x
$$

A direct calculation using (5.21), (5.24) and the Bramble-Hilbert lemma (cf. [6, 19]) shows that

$$
h_{k}^{2} \sum_{p \in \mathcal{V}_{k} \cap \bar{\Omega}_{q}} n(p)\left[v(p)-v_{q}\right]^{2} \leq c_{2}\left\|v-v_{q}\right\|_{L_{2}\left(\Omega_{q}\right)}^{2} \leq c_{3} \ell^{2} h_{k}^{2}|v|_{H^{1}\left(\Omega_{q}\right)}^{2} .
$$


Using (5.5) we have

$$
\sum_{p \in \mathcal{V}_{k} \cap \bar{\Omega}_{q}} n(p)=(n(q)-1)+\sum_{p \in \mathcal{V}_{k} \cap \bar{\Omega}_{q}} 1 \leq\left(1+c_{4} \ell^{-2}\right) \sum_{p \in \mathcal{V}_{k} \cap \bar{\Omega}_{q}} 1 .
$$

We find from (5.23), (5.25) and (5.26) that

$$
h_{k}^{2} \sum_{p \in \mathcal{V}_{k} \cap \bar{\Omega}_{q}} n(p)[v(p)]^{2} \leq\left(1+c_{5} \ell^{-2}\right) h_{k}^{2}\left(\sum_{p \in \mathcal{V}_{k} \cap \bar{\Omega}_{q}} v_{q}^{2}\right)+c_{6} \ell^{4} h_{k}^{2}|v|_{H^{1}\left(\Omega_{q}\right)}^{2} .
$$

On the other hand we also have, by (5.25),

$$
\begin{aligned}
h_{k}^{2} \sum_{p \in \mathcal{V}_{k} \cap \bar{\Omega}_{q}} v_{q}^{2} & \leq\left(1+\ell^{-2}\right) h_{k}^{2} \sum_{p \in \mathcal{V}_{k} \cap \bar{\Omega}_{q}}[v(p)]^{2}+\left(1+\ell^{2}\right) h_{k}^{2} \sum_{p \in \mathcal{V}_{k} \cap \bar{\Omega}_{q}}\left[v_{q}-v(p)\right]^{2} \\
& \leq\left(1+\ell^{-2}\right) h_{k}^{2} \sum_{p \in \mathcal{V}_{k} \cap \bar{\Omega}_{q}}[v(p)]^{2}+c_{7} \ell^{4} h_{k}^{2}|v|_{H^{1}\left(\Omega_{q}\right)}^{2} .
\end{aligned}
$$

Combining (5.27) and (5.28) we obtain

$$
h_{k}^{2} \sum_{p \in \mathcal{V}_{k} \cap \bar{\Omega}_{q}} n(p)[v(p)]^{2} \leq\left(1+c_{8} \ell^{-2}\right) h_{k}^{2} \sum_{p \in \mathcal{V}_{k} \cap \bar{\Omega}_{q}}[v(p)]^{2}+c_{9} \ell^{4} h_{k}^{2}|v|_{H^{1}\left(\Omega_{q}\right)}^{2} .
$$

The estimate (5.19) is established by summing up (5.22) and (5.29) (over all $\left.q \in \mathcal{V}_{1}\right)$.

Lemma 5.6. There exists a positive constant $C$ such that

$$
\begin{aligned}
\|v\|_{1-\beta, k}^{2} \leq\left(1+\theta^{2}\right)\|v\|_{1-\beta, k}^{2}+C \theta^{-4} h_{k}^{2 \beta}\|v\|_{a}^{2} & \forall \theta \in(0,1], v \in V_{k}, \\
\|v\|_{1-\beta, k}^{2} \leq\left(1+\theta^{2}\right)\|v\|_{1-\beta, k}^{2}+C \theta^{-4} h_{k}^{2 \beta}\|v\|_{a}^{2} & \forall \theta \in(0,1], v \in V_{k} .
\end{aligned}
$$

Proof. The estimates (4.2), (4.4) and (5.18) imply that there exists a positive constant $C$ such that

$$
\|v\|_{0, k}^{2} \leq\left(1+\theta^{2}\right)\|v\|_{0, k}^{2}+C \theta^{-4} h_{k}^{2 \beta}\|v\|_{\beta, k}^{2} \quad \forall v \in V_{k} .
$$

On the other hand it follows from (4.2) and (5.9) that

$$
\|v\|_{1, k}^{2}=\|v\|_{1, k}^{2} \leq\left(1+\theta^{2}\right)\|v\|_{1, k}^{2}+C \theta^{-4} h_{k}^{2 \beta}\|v\|_{1+\beta, k}^{2} \quad \forall v \in V_{k} .
$$

The estimate (5.31) follows from interpolating (5.32) and (5.33) between the

Hilbert scales $\left(V_{k},\|\| \cdot\|\|_{s, k}\right)$ and $\left(V_{k}, \sqrt{\left(1+\theta^{2}\right)\|\cdot\|_{s, k}^{2}+C \theta^{-4} h_{k}^{2 \beta}\|\cdot\|_{s+\beta, k}^{2}}\right)$.

The proof of (5.30) is similar.

The estimates (5.1) and (5.2) follow from Lemma 5.2, Lemma 5.3 and Lemma 5.6

\section{Convergence analysis of the $V$-CyCle algorithm}

Let us first introduce some operators that will simplify many expressions in the convergence analysis. For $2 \leq j<k$, the operator $T_{k, j, m}: V_{j} \longrightarrow V_{k}$ is defined by

$$
T_{k, j, m}=R_{k}^{m} R_{k-1}^{m} \cdots R_{j+1}^{m},
$$

and the operator $T_{j, k, m}: V_{k} \longrightarrow V_{j}$ defined by

$$
T_{j, k, m}=P_{j} R_{j+1}^{m} \cdots P_{k-1} R_{k}^{m}
$$

is the transpose of $T_{k, j, m}$ with respect to the variational form $a(\cdot, \cdot)$. The operator $T_{k, k, m}$ is defined to be the identity operator $I d_{k}$ on $V_{k}$. 
Remark 6.1. It is more precise to write

$$
T_{k, j, m}=R_{k}^{m} I_{k-1}^{k} R_{k-1}^{m} I_{k-2}^{k-1} \cdots R_{j+1}^{m} I_{j}^{j+1},
$$

where $I_{\ell}^{\ell+1}: V_{\ell} \longrightarrow V_{\ell+1}$ is the natural injection. Such natural injections have been suppressed in Section 2 and (6.1).

Let $K \geq 2$ be an arbitrary but fixed integer and

$$
v_{k}=\left(P_{k}-P_{k-1}\right) v \quad \forall v \in V_{K}, 2 \leq k<K .
$$

Note that the $v_{k}$ 's are pairwise orthogonal with respect to $a(\cdot, \cdot)$, and

$$
\sum_{k=2}^{K}\left\|v_{k}\right\|_{a}^{2}=\left\|v-P_{1} v\right\|_{a}^{2} \leq\|v\|_{a}^{2} .
$$

Moreover, we have

$$
v_{k}=\left(I d_{k}-P_{k-1}\right) v_{k} .
$$

We can write, by (2.27) and (6.1)-(6.3),

$$
\begin{aligned}
& a\left(E_{K} v, E_{K} v\right) \\
= & \sum_{j, k=2}^{K} a\left(R_{K}^{m} \cdots R_{j+1}^{m} R_{j}^{m}\left(P_{j}-P_{j-1}\right) v, R_{K}^{m} \cdots R_{k+1}^{m} R_{k}^{m}\left(P_{k}-P_{k-1}\right) v\right) \\
= & \sum_{k=2}^{K} a\left(R_{k}^{m} v_{k}, T_{k, K, m} T_{K, k, m} R_{k}^{m} v_{k}\right) \\
& +2 \sum_{2 \leq j<k \leq K} a\left(R_{j}^{m} v_{j}, T_{j, k, m} T_{k, K, m} T_{K, k, m} R_{k}^{m} v_{k}\right) .
\end{aligned}
$$

The properties of the operators $T_{j, k, m}$ are therefore crucial for the convergence analysis. From (2.14) and (4.9) we have, for $j, k \geq 2$, the trivial estimate

$$
\left\|T_{j, k, m} v\right\|_{1, j} \leq\|v\|_{1, k} \quad \forall v \in V_{k} .
$$

It follows from (4.2), (4.10), (6.5) and (6.7) that

$$
a\left(R_{k}^{m} v_{k}, T_{k, K, m} T_{K, k, m} R_{k}^{m} v_{k}\right) \leq\left\|R_{k}^{m} v_{k}\right\|_{1, k}^{2} \lesssim m^{-\alpha}\left\|v_{k}\right\|_{a}^{2} .
$$

In order to estimate the remaining terms on the right-hand side of ([6.6]) we need some less trivial properties of the operators $T_{j, k, m}$. We begin the study of these properties with a lemma on the Ritz projection operators.

Lemma 6.2. There exists a positive constant $C$ such that

$$
\left\|P_{k-1} v\right\|_{1-\beta, k-1}^{2} \leq\left(1+\theta^{2}\right)\|v\|_{1-\beta, k}^{2}+C \theta^{-4} h_{k}^{2 \beta}\|v\|_{a}^{2}
$$

$\forall \theta \in(0,1], v \in V_{k}$.

Proof. It follows from (2.14) and (5.2) that

$$
\begin{aligned}
\left\|P_{k-1} v\right\|_{1-\beta, k-1}^{2} \leq & \left(1+\theta^{2}\right)\left\|P_{k-1} v\right\|_{1-\beta, k}^{2}+c_{1} \theta^{-4} h_{k}^{2 \beta}\left\|P_{k-1} v\right\|_{a}^{2} \\
\leq & \left(1+c_{2} \theta^{2}\right)\|v\|_{1-\beta, k}^{2} \\
& +\left[c_{3} \theta^{-2}\left\|P_{k-1} v-v\right\|_{1-\beta, k}^{2}+c_{1} \theta^{-4} h_{k}^{2 \beta}\|v\|_{a}^{2}\right] .
\end{aligned}
$$

The estimate (6.9) follows from (2.2), (3.6), (4.7) and (6.10).

The properties of the operators $T_{j, k, m}$ are given in the next three lemmas. 
Lemma 6.3. The following estimate holds:

$$
\left\|T_{k, K, m} v\right\|_{1-\beta, k} \lesssim\|v\|_{1-\beta, K}+h_{k}^{\beta}\|v\|_{a} \quad \forall v \in V_{K}, 2 \leq k \leq K .
$$

Proof. The case where $k=K$ is trivial. For $2 \leq k<K$, it follows from (2.2), (4.9), (6.2), (6.7) and (6.9) that, for any $v \in V_{K}$,

$$
\begin{aligned}
& \left\|T_{k, K, m} v\right\|_{1-\beta, k}^{2} \\
& \quad=\left\|P_{k} R_{k+1}^{m} T_{k+1, K, m} v\right\|_{1-\beta, k}^{2} \\
& \quad \leq\left(1+\theta_{k}^{2}\right)\left\|R_{k+1}^{m} T_{k+1, K, m} v\right\|_{1-\beta, k+1}^{2}+c_{*} \theta_{k}^{-4} h_{k}^{2 \beta}\left\|R_{k+1}^{m} T_{k+1, K, m} v\right\|_{a}^{2} \\
& \quad \leq\left(1+\theta_{k}^{2}\right)\left\|T_{k+1, K, m} v\right\|_{1-\beta, k+1}^{2}+c_{*} \theta_{k}^{-4} h_{k}^{2 \beta}\|v\|_{a}^{2},
\end{aligned}
$$

where $\theta_{k} \in(0,1]$ is arbitrary, and $c_{*}$ is independent of the meshes and $\theta_{k}$.

Iterating (6.12) we find

$$
\begin{aligned}
\left\|T_{k, K, m} v\right\|_{1-\beta, k}^{2} \leq\left[\prod_{\ell=k}^{K-1}(1+\right. & \left.\left.\theta_{\ell}^{2}\right)\right]\|v\|_{1-\beta, K}^{2} \\
& +c_{*}\left[\sum_{\ell=k}^{K-1} h_{\ell}^{2 \beta} \theta_{\ell}^{-4} \prod_{r=k}^{\ell-1}\left(1+\theta_{r}^{2}\right)\right]\|v\|_{a}^{2},
\end{aligned}
$$

where the $\theta_{\ell}$ 's $(k \leq \ell \leq K-1)$ are arbitrary numbers in $(0,1]$.

By taking $\theta_{\ell}=3^{-\beta(\ell-k) / 4}$, we deduce from (2.2) and (6.13) that

$$
\left\|T_{k, K, m} v\right\|_{1-\beta, k}^{2} \leq \omega\|v\|_{1-\beta, K}^{2}+c_{*} \omega \eta h_{k}^{2 \beta}\|v\|_{a}^{2} \quad \forall v \in V_{K},
$$

where

$$
\omega=\prod_{n=0}^{\infty}\left(1+3^{-(\beta / 2) n}\right) \quad \text { and } \quad \eta=\sum_{n=0}^{\infty}\left(\frac{3}{4}\right)^{\beta n} .
$$

The estimate (6.11) follows from (6.14) and (6.15).

Lemma 6.4. The following estimate holds:

$$
\left\|T_{K, k, m} v\right\|_{1-\beta, K} \lesssim\|v\|_{1-\beta, k} \quad \forall v \in V_{k}, 2 \leq k \leq K .
$$

Proof. The case where $k=K$ is trivial. For $2 \leq k<K$, using (4.9), (5.1), (6.1) and (6.7) we obtain

$$
\begin{aligned}
\left\|T_{K, k, m} v\right\|_{1-\beta, K}^{2} & =\left\|R_{K}^{m} T_{K-1, k, m} v\right\|_{1-\beta, K}^{2} \\
& \leq\left\|T_{K-1, k, m} v\right\|_{1-\beta, K}^{2} \\
& \leq\left(1+\theta_{K-1}^{2}\right)\left\|T_{K-1, k, m} v\right\|_{1-\beta, K-1}^{2}+c_{*} \theta_{K-1}^{-4} h_{K-1}^{2 \beta}\|v\|_{a}^{2}
\end{aligned}
$$

$\forall v \in V_{k}$, where $\theta_{K-1} \in(0,1]$ is arbitrary and the positive constant $c_{*}$ is independent of the meshes and $\theta_{K}$.

By iterating (6.17) we find

$$
\begin{aligned}
&\left\|T_{K, k, m} v\right\|_{1-\beta, K}^{2} \leq c_{*} {\left[\sum_{\ell=k}^{K-1} h_{\ell}^{2 \beta} \theta_{\ell}^{-4} \prod_{\ell+1 \leq r \leq K-1}\left(1+\theta_{r}^{2}\right)\right]\|v\|_{a}^{2} } \\
&+\left[\prod_{\ell=k}^{K-1}\left(1+\theta_{\ell}^{2}\right)\right]\|v\|_{1-\beta, k}^{2} \quad \forall v \in V_{k},
\end{aligned}
$$

where $\theta_{k}, \ldots, \theta_{K-1} \in(0,1]$ are arbitrary. 
As in the proof of Lemma 6.3, by taking $\theta_{\ell}=3^{-\beta(\ell-k) / 4}$ for $k \leq \ell \leq K-1$, we obtain from (6.18) the estimate

$$
\left\|T_{K, k, m} v\right\|_{1-\beta, K}^{2} \lesssim\|v\|_{1-\beta, k}^{2}+h_{k}^{2 \beta}\|v\|_{a}^{2} \quad \forall v \in V_{k},
$$

which together with (4.2) and (4.4) imply (6.16).

Lemma 6.5. The following estimate holds:

$$
\left\|T_{k, K, m} T_{K, k, m} v\right\|_{1-\beta, k} \lesssim\|v\|_{1-\beta, k} \quad \forall v \in V_{k}, 2 \leq k \leq K .
$$

Proof. From (4.2), (4.4), (6.7), (6.11) and (6.16) we have, for any $v \in V_{k}$,

$$
\begin{aligned}
\left\|T_{k, K, m} T_{K, k, m} v\right\|_{1-\beta, k} & \lesssim\left\|T_{K, k, m} v\right\|_{1-\beta, K}+h_{k}^{\beta}\left\|T_{K, k, m} v\right\|_{a} \\
& \lesssim\|v\|_{1-\beta, k}+h_{k}^{\beta}\|v\|_{a} \\
& \lesssim\|v\|_{1-\beta, k} .
\end{aligned}
$$

Lemma 6.6. Let $2 \leq j<k$ and $\theta \in(0,1]$. There exists a mesh-independent positive integer $m_{\theta}$ such that

$$
\left\|T_{j, k, m} v\right\|_{1-\beta, j} \leq\left(1+2 \theta^{2}\right)^{(k-j) / 2}\|v\|_{1-\beta, k} \quad \forall v \in V_{k}, m \geq m_{\theta} .
$$

Proof. From (4.2), (4.8), (4.9), (6.2) and (6.9), we have

$$
\begin{aligned}
\left\|T_{j, k, m} v\right\|_{1-\beta, j}^{2} & =\left\|P_{j} R_{j+1}^{m} T_{j+1, k, m} v\right\|_{1-\beta, j}^{2} \\
& \leq\left(1+\theta^{2}\right)\left\|R_{j+1}^{m} T_{j+1, k, m} v\right\|_{1-\beta, j+1}^{2}+C \theta^{-4} h_{j+1}^{2 \beta}\left\|R_{j+1}^{m} T_{j+1, k, m} v\right\|_{a}^{2} \\
& \leq\left(1+\theta^{2}\right)\left\|T_{j+1, k, m} v\right\|_{1-\beta, j+1}^{2}+C m^{-\beta} \theta^{-4}\left\|T_{j+1, k, m} v\right\|_{1-\beta, j+1}^{2},
\end{aligned}
$$

where $C$ is independent of the meshes and $\theta$.

Therefore, by choosing $m_{\theta}$ large enough, we have

$$
\left\|T_{j, k, m} v\right\|_{1-\beta, j}^{2} \leq\left(1+2 \theta^{2}\right)\left\|T_{j+1, k, m} v\right\|_{1-\beta, j+1}^{2} \quad \forall v \in V_{k}, m \geq m_{\theta} .
$$

The estimate (6.20) follows by iterating (6.21).

Given any $\theta \in(0,1]$, we can now estimate the terms in the second sum on the right-hand side of (6.6) by using (2.2), (4.6), (4.8), Lemma 6.5 and Lemma 6.6 as follows.

For $2 \leq j<k \leq K$, we have

$$
\begin{aligned}
& a\left(R_{j}^{m} v_{j}, T_{j, k, m} T_{k, K, m} T_{K, k, m} R_{k}^{m} v_{k}\right) \\
\leq & \left\|R_{j}^{m} v_{j}\right\|_{1+\beta, j}\left\|T_{j, k, m} T_{k, K, m} T_{K, k, m} R_{k}^{m} v_{k}\right\|_{1-\beta, j} \\
\leq & C\left(1+2 \theta^{2}\right)^{(k-j) / 2}\left\|R_{j}^{m} v_{j}\right\|_{1+\beta, j}\left\|R_{k}^{m} v_{k}\right\|_{1-\beta, k} \\
\leq & C m^{-\beta}\left(1+2 \theta^{2}\right)^{(k-j) / 2}\left(\frac{h_{k}}{h_{j}}\right)^{\beta}\left(h_{j}^{-\beta}\left\|R_{j}^{\lceil m / 2\rceil} v_{j}\right\|_{1-\beta, j}\right) \\
& \times\left(h_{k}^{-\beta}\left\|R_{k}^{m} v_{k}\right\|_{1-\beta, k}\right) \\
\leq & C m^{-\beta}\left[\left(1+2 \theta^{2}\right)^{1 /(2 \beta)} 2^{-1}\right]^{\beta(k-j)}\left(h_{j}^{-\beta}\left\|R_{j}^{\lceil m / 2\rceil} v_{j}\right\|_{1-\beta, j}\right) \\
& \times\left(h_{k}^{-\beta}\left\|R_{k}^{m} v_{k}\right\|_{1-\beta, k}\right),
\end{aligned}
$$

where $m \geq m_{\theta}$ for a sufficiently large $m_{\theta}$.

The following strengthened Cauchy-Schwarz inequality provides the last key estimate for the convergence analysis. 
Lemma 6.7. Let $v \in V_{K}$ be arbitrary and $v_{k}(2 \leq k \leq K)$ be defined by (6.3). There exists a positive integer $M$ independent of the meshes and $v$ such that

$$
a\left(R_{j}^{m} v_{j}, T_{j, k, m} T_{k, K, m} T_{K, k, m} R_{k}^{m} v_{k}\right) \lesssim m^{-\alpha}\left(\frac{2}{3}\right)^{\beta(k-j)}\left\|v_{j}\right\|_{a}\left\|v_{k}\right\|_{a}
$$

for $2 \leq j<k \leq K$ and $m \geq M$.

Proof. Combining (4.10), (6.5) and (6.22), we find

$$
\begin{aligned}
& a\left(R_{j}^{m} v_{j}, T_{j, k, m} T_{k, K, m} T_{K, k, m} R_{k}^{m} v_{k}\right) \\
& \quad \leq C m^{-\beta}\left[\left(1+2 \theta^{2}\right)^{1 /(2 \beta)} 2^{-1}\right]^{\beta(k-j)}\left(m^{(\beta-\alpha) / 2}\left\|v_{j}\right\|_{a}\right)\left(m^{(\beta-\alpha) / 2}\left\|v_{k}\right\|_{a}\right) \\
& \quad \leq C m^{-\alpha}\left[\left(1+2 \theta^{2}\right)^{1 /(2 \beta)} 2^{-1}\right]^{\beta(k-j)}\left\|v_{j}\right\|_{a}\left\|v_{k}\right\|_{a} \quad \forall m \geq m_{\theta} .
\end{aligned}
$$

The estimate (6.23) follows by choosing a small $\theta$ so that $\left(1+2 \theta^{2}\right)^{1 /(2 \beta)} 2^{-1}<\frac{2}{3}$.

We are now ready to prove the main results of this paper.

Lemma 6.8. There exists a positive integer $M$ such that

$$
\left\|E_{K, m} v\right\|_{a} \lesssim m^{-\alpha / 2}\|v\|_{a} \quad \forall v \in V_{K}, m \geq M, K=1,2,3, \ldots
$$

Proof. The case $K=1$ is trivial since $E_{1, m}=0$. For $K \geq 2$, we have, by (6.4), (6.6), (6.8), (6.23) and the discrete Young's inequality (cf. [24]),

$$
\begin{aligned}
\left\|E_{K, m} v\right\|_{a}^{2}=a\left(E_{K, m} v, E_{K, m} v\right) & \lesssim m^{-\alpha} \sum_{j, k=2}^{K}\left(\frac{2}{3}\right)^{\beta|k-j|}\left\|v_{j}\right\|_{a}\left\|v_{k}\right\|_{a} \\
& \lesssim m^{-\alpha}\left[\sum_{n=0}^{\infty}\left(\frac{2}{3}\right)^{\beta n}\right] \sum_{k=2}^{K}\left\|v_{k}\right\|_{a}^{2} \\
& \lesssim m^{-\alpha} \sum_{k=2}^{K}\left\|v_{k}\right\|_{a}^{2} \lesssim m^{-\alpha}\|v\|_{a}^{2} .
\end{aligned}
$$

As a corollary to Lemma 6.8 and the result (1.15), we have an error estimate for the $V$-cycle algorithm with any number of smoothing steps.

Theorem 6.9. Given any $g \in V_{k}$ and any initial guess $z_{0} \in V_{k}$, the approximate solution $M G_{\mathcal{V}}\left(k, g, z_{0}, m_{1}, m_{2}\right)$ of (1.12) obtained by Algorithm 2.1 satisfies the error estimate

$$
\begin{aligned}
\| z- & M G_{\mathcal{V}}\left(k, g, z_{0}, m_{1}, m_{2}\right) \|_{a} \\
\leq & {\left[\frac{C}{C+\left[\max \left(m_{1}, 1\right) \max \left(m_{2}, 1\right)\right]^{\alpha / 2}}\right]\left\|z-z_{0}\right\|_{a} }
\end{aligned}
$$

for $m_{1}+m_{2} \geq 1$, where the positive constant $C$ is mesh independent.

Proof. It follows from (1.15) that

$$
\left\|E_{k, m} v\right\|_{a} \leq \delta\|v\|_{a} \quad \forall v \in V_{k}, k=1,2, \ldots,
$$

where $\delta \in(0,1)$ is independent of the meshes and the number of smoothing steps. 
From Lemma 6.8 we have

$$
\left\|E_{k, m} v\right\|_{a} \leq \tilde{C} m^{-\alpha / 2}\|v\|_{a} \quad \forall v \in V_{k}, m \geq M, k=1,2, \ldots
$$

Let $M_{*} \geq M$ be chosen large enough so that

$$
\tilde{C} m^{-\alpha / 2} \leq \frac{2 \tilde{C}}{2 \tilde{C}+m^{\alpha / 2}} \quad \forall m \geq M_{*}+1,
$$

and then let $C$ be chosen large enough so that

$$
C \geq 2 \tilde{C} \quad \text { and } \quad \delta \leq \frac{C}{C+M_{*}^{\alpha / 2}} .
$$

It follows from (6.26)- 6.29 that

$$
\left\|E_{k, m} v\right\|_{a} \leq\left[\frac{C}{C+m^{\alpha / 2}}\right]\|v\|_{a} \quad \forall v \in V_{k}, k=1,2, \ldots,
$$

i.e., the estimate (6.25) holds for $m_{1}=0$ and $m_{2}=m$.

The general case of (6.25) follows easily from this special case and the relations (2.24) and (2.25).

Remark 6.10. The results of this paper can be easily generalized to two- and threedimensional elliptic boundary value problems discretized by the $\mathrm{Q}_{1}$ element.

Remark 6.11. In the case where $\Omega$ also has cracks, the elliptic regularity estimate is valid for $0<\alpha<\frac{1}{2}$ ( $\alpha$ can be taken to be arbitrarily close to $\frac{1}{2}$, cf. [21, 18, 27]). All the statements in this paper remain valid provided the concept of Sobolev spaces on cracked domains are defined appropriately (cf. [15]). In particular, Theorem 6.9 also holds for domains with cracks.

Remark 6.12. The new approach to $V$-cycle convergence analysis developed in this paper can also be applied to nonconforming finite elements. This will be addressed in a forthcoming paper.

Acknowledgments. The author would like to thank Dietrich Braess and an anonymous referee for many constructive comments on an earlier version of this paper.

\section{REFERENCES}

[1] R.E. Bank and C.C. Douglas, Sharp estimates for multigrid rates of convergence with general smoothing and acceleration, SIAM J. Numer. Anal. 22 (1985), 617-633. MR 86j:65037

[2] R.E. Bank and T.F. Dupont, An optimal order process for solving finite element equations, Math. Comp. 36 (1981), 35-51. MR 82b:65113

[3] C. Bennett and R. Sharpley, Interpolation of operators, Academic Press, Boston, 1988. MR 89e:46001

[4] D. Braess and W. Hackbusch, A new convergence proof for the multigrid method including the V-cycle, SIAM J. Numer. Anal. 20 (1983), 967-975. MR 85h:65233

[5] J.H. Bramble, Multigrid Methods, Pitman Research Notes in Mathematics Series 294, Longman Scientific \& Technical, New York, 1993. MR 95b:65002

[6] J.H. Bramble and S.R. Hilbert, Estimation of linear functionals on Sobolev spaces with application to Fourier transforms and spline interpolation, SIAM J. Numer. Anal. 7 (1970), 113-124. MR 41:7819

[7] J.H. Bramble and J.E. Pasciak, New convergence estimates for multigrid algorithms, Math. Comp. 49 (1987), 311-329. MR 89b:65234

[8] - New estimates for multigrid algorithms including the V-cycle, Math. Comp. 60 (1993), 447-471. MR 94a:65064 
[9] _ Uniform convergence estimates for multigrid $V$-cycle algorithms with less than full elliptic regularity, Domain Decomposition Methods in Science and Engineering (Como, 1992), Contemp. Math. 157, Amer. Math. Soc., Providence, RI, 1994, pp. 17-26. MR 95f:65202

[10] J.H. Bramble and X. Zhang, The analysis of multigrid methods, Handb. Numer. Anal. VII, North-Holland, Amsterdam, 2000, 173-415. CMP 2001:08

[11] Uniform convergence of the multigrid $V$-cycle for an anisotropic problem, Math. Comp. 70 (2001), 453-470.

[12] S.C. Brenner, Multigrid methods for the computation of singular solutions and stress intensity factors I: Corner singularities, Math. Comp. 68 (1999), 559-583. MR 99i:65138

[13] _ A nonstandard finite element interpolation estimate, Numer. Funct. Anal. Optim. 20 (1999), 245-250. MR 2000b:65210

[14] S.C. Brenner and L.R. Scott, The mathematical theory of finite element methods, SpringerVerlag, New York-Berlin-Heidelberg, 1994. MR 95f:65001

[15] S.C. Brenner and L.-Y. Sung, Multigrid methods for the computation of singular solutions and stress intensity factors II: Crack singularities, BIT 37 (1997), 623-643. MR 99i:65139

[16] P.-G. Ciarlet, The Finite Element Method for Elliptic Problems, North Holland, AmsterdamNew York-Oxford, 1978. MR 58:25001

[17] P. Clément, Approximation by finite element functions using local regularization, R.A.I.R.O. Sér. Rouge Anal. Numér. 9 (1975), 77-84. MR 53:4569

[18] M. Dauge, Elliptic boundary value problems on corner domains (Lecture Notes in Mathematics 1341), Springer-Verlag, Berlin-Heidelberg, 1988. MR 91a:35078

[19] T. Dupont and R. Scott, Polynomial approximation of functions in Sobolev spaces, Math. Comp. 34 (1980), 441-463. MR 81h:65014

[20] M. Griebel and P. Oswald, On the abstract theory of additive and multiplicative Schwarz algorithms, Numer. Math. 70 (1995), 163-180. MR 96a:65164

[21] P. Grisvard, Elliptic problems in nonsmooth domains, Pitman, Boston, 1985. MR 86m:35044

[22] W. Hackbusch, Multigrid convergence theory, Proceedings of the Conference Held at KölnPorz, November 23-27, 1981 (W. Hackbusch and U. Trottenberg, eds.), Lecture Notes in Mathematics 960, Springer-Verlag, Berlin, 1982, pp. 177-219. MR 84k:65113

[23] _ Multigrid methods and applications, Springer-Verlag, Berlin, 1985. MR 87e:65082

[24] G.H. Hardy, J.E. Littlewood and G. Pólya, Inequalities, Cambridge University Press, Cambridge, 1934.

[25] J. Mandel, S. McCormick and R. Bank, Variational multigrid theory, Multigrid Methods (S.F. McCormick, ed.), Frontiers In Applied Mathematics 3, SIAM, Philadelphia, 1987, pp. 131-177. CMP 21:05

[26] S.F. McCormick, Multigrid methods for variational problems: further results, SIAM J. Numer. Anal. 21 (1984), 255-263. MR 85h:65115

[27] S.A. Nazarov and B.A. Plamenevsky, Elliptic problems in domains with piecewise smooth boundaries, Expositions in Mathematics 13, de Gruyter, Berlin-New York, 1994. MR 95h:35001

[28] N. Neuss, $V$-cycle convergence with unsymmetric smoothers and applications to an anisotropic model problem, SIAM J. Numer. Anal. 35 (1998), 1201-1212.

[29] L.R. Scott and S. Zhang, Finite element interpolation of nonsmooth functions satisfying boundary conditions, Math. Comp. 54 (1990), 483-493. MR 90j:65021

[30] H. Triebel, Interpolation theory, function spaces, differential operators, North-Holland, Amsterdam, 1978. MR 80i:46032b

[31] J. Xu, Iterative methods by space decomposition and subspace correction, SIAM Review 34 (1992), 581-613. MR 93k:65029

[32] H. Yserentant, Old and new convergence proofs for multigrid methods, Acta Numer. (1993), 285-326. MR 94i:65128

[33] X. Zhang, Multilevel Schwarz methods, Numer. Math. 63 (1992), 521-539. MR 93h:650247

Department of Mathematics, University of South Carolina, Columbia, South CarOLINA 29208

E-mail address: brenner@math.sc.edu 